\title{
Une coopérative financière au service du milieu : l'innovation dans la culture
}

\author{
Martine Vézina, HEC Montréal \\ Damien Rousselière, Université Pierre Mendès France (Grenoble)
}

\section{Introduction}

C et article a pour objectif de présenter une étude empirique montrant comment la forme coopérative peut constituer une réponse aux enjeux spécifiques d'un milieu, qu'il soit territorialisé ou non. C'est à travers l'analyse du modèle de développement de la Caisse Desjardins de la culture que nous nous proposons de poursuivre cet objectif.

Cette étude s'articulant donc autour de la question du « comment», notre analyse s'appuie sur une approche stratégique issue de l'approche des ressources (Resource-Based View) ${ }^{1}$. L'approche des ressources stipule que les raisons du succès (performance) des organisations tiennent principalement à la combinaison originale qu'elles font des actifs (tangibles et intangibles) qu'elles détiennent et des processus organisationnels qu'elles élaborent au cours de leur développement. À cet égard, l'approche des ressources s'intéresse moins à la place qu'occupe l'organisation dans son environnement concurrentiel qu'au soin qu'elle met à développer un modèle organisationnel approprié à sa stratégie (activités et marchés desservis). Parce que nous nous intéressons au développement des milieux, notre étude empirique s'appuie sur une organisation coopérative financière, la Caisse Desjardins de la culture. On sait, en effet, que le financement est un levier majeur du développement des organisations, mais également des milieux. S'intéresser aux raisons de la performance d'une coopérative financière, c'est donc chercher à comprendre sa contribution directe à ses membres, mais également au milieu dans lequel elle évolue.

Dans un premier temps, nous faisons état des spécificités du milieu culturel et des besoins qui en dé- coulent en termes de financement de l'activité. Nous abordons ensuite plus particulièrement le modèle de la Caisse de la culture et ses diverses modalités d'intervention, mettant ainsi l'accent sur ce qui nous semble être au fondement de son succès : l'accompagnement dans la relation de service, l'appui du réseau partenarial ainsi que le développement de méthodes d'analyse et de gestion du risque adaptées. En conclusion, nous proposons quelques pistes de réflexion quant aux conditions par lesquelles une institution financière est susceptible de participer au développement de son milieu.

\section{L'émergence de la Caisse de la culture : une initiative du milieu}

Il est nécessaire de replacer la Caisse de la culture dans le cadre spécifique du secteur culturel, qui se caractérise par des statuts divers, une répartition particulière des revenus et des besoins importants de financement. Ces problèmes touchent de manière différenciée les diverses acteurs de ce secteur, suivant qu'ils sont travailleurs autonomes, entrepreneurs individuels ou entreprises culturelles.

Ainsi, du point de vue du travail autonome, le constat de l'existence d'une « économie du star-system » est assez récurrent. Cette concentration importante des gains sur un nombre relativement limité d'acteurs et la forte précarité des autres apparaît notamment dans le rapport récent du ministère de la Culture et des Communications du Québec sur la situation socio-économique des artistes : leur situation se caractérise par une très forte fluctuation des revenus d'une année sur l'autre et une forte inégalité de la répartition de ces mêmes revenus ${ }^{2}$. 
Les besoins de financement découlant de cette situation de précarité sont importants et à ne pas négliger pour les différentes organisations professionnelles d'artistes. Celles-ci se sont toujours préoccupées du problème du financement pour leurs membres, pour des besoins personnels ou professionnels.

\section{La création de la Caisse de la culture apparaît comme une réponse partielle à ces problèmes de financement de l'économie de la culture.}

La spécificité du financement se pose également du point de vue de l'entreprise culturelle. La nature de service ou de bien immatériel pose un problème important de coûts noyés, c'est-à-dire des coûts fixes engagés avant la réalisation du produit final. D'autre part, son caractère de prototype questionne la façon d'évaluer la qualité de ce bien et le risque de débouché qui y est associé. Dans ce contexte, ces entreprises doivent faire face à une multiplicité de sources de financement (public, dons et commandites, ventes...) plus ou moins récurrentes et souvent précaires. La faiblesse des débouchés locaux les oblige à tenter de trouver des moyens d'élargir le marché, et donc à aller vers les marchés étrangers, francophones ou américains.

La création de la Caisse de la culture apparaît comme une réponse partielle à ces problèmes de financement de l'économie de la culture. Sa création, en 1994, est liée à un contexte politique de forte volonté d'autonomisation du secteur québécois de la culture. En effet, la structuration de ce secteur culturel s'est traduite par la mise en place progressive de 1988 à 1997 d'un ensemble d'institutions impliquant la reconnaissance spécifique du rôle des partenaires sociaux dans une régulation multipolaire de la culture ${ }^{3}$.

La discussion sur la nouvelle politique culturelle du Québec de 1992 a contribué à la création de nombreux réseaux consolidant le secteur culturel. Ces réseaux inscrivent également leur réflexion dans un rapport particulier à la place de la culture québécoise. Porté par un acteur déterminant dans cette période et fort de sa légitimité, l'Union des artistes (UDA), le projet de caisse est directement issu d'un rapprochement entre les différentes organisations professionnelles du secteur culturel : l'UDA, l'UNEQ (Unions des écrivaines et écrivains du Québec), la GMMQ (Guil- de des musiciennes et musiciens du Québec), les autres centrales syndicales et organisations professionnelles, et le mouvement Desjardins.

Dans un contexte de forte restructuration et de rationalisation de son réseau de caisses, le mouvement Desjardins est toutefois peu enclin à créer une nouvelle entité. Le projet part donc plutôt d'une caisse existante à l'ONF (Office national du film) en en changeant la structure organisationnelle. Renouvelée, cette caisse comptera initialement 400 membres avec un conseil d'administration de 12 personnes (dont 3 membres de l'ancienne communauté). Les 9 autres allaient être des individus membres de l'UDA. La nouvelle structure, qu'on appellerait dorénavant la Caisse de la culture, fut lancée le 19 avril 1994, avec un actif de 1,5 million de dollars. Elle se dote alors d'une mission très claire : «Utiliser l'argent du milieu pour développer le milieu ». Quelle autre mission pourrait-elle de toute façon poursuivre compte tenu du fait qu'elle émane de façon très forte de ce milieu qu'est celui de la culture?

En 11 ans, la Caisse connaît un développement important de son activité. Les actifs (au bilan) passent de 1 million \$ à plus de 80 millions \$, alors que le membership, maintenant de 3900 personnes, progresse d'à peu près $10 \%$ par an depuis sa fondation. Même si la Caisse de la culture est d'envergure provinciale, $70 \%$ de l'activité économique reliée à la culture se passant à Montréal (30\% en région), plus de $90 \%$ de ses membres sont de la région métropolitaine. Les secteurs culturels représentés dans la clientèle sont variés, mais la portion de membres issus de l'UDA est encore prédominante, comptant pour près de la moitié des membres de la Caisse. La Caisse de la culture compte aussi des sociétaires parmi les membres de la GMMQ, de l'UNEQ, du personnel de l'ONF et surtout du personnel de l'UQAM (Université du Québec à Montréal) (près de 1 100). La création d'un point de service à l'UQAM peut aussi se voir dans un même mouvement visant à accentuer le rythme de croissance de la Caisse et visant à la solidifier financièrement. Avec le temps, la clientèle s'est également élargie à l'ensemble des secteurs d'activités touchant le monde de la culture et des communications. Ainsi, des danseurs, des sculpteurs, des peintres et même des journalistes, des producteurs et des techniciens du monde du spectacle sont maintenant parmi les membres de la Caisse de la culture et y représentent environ $5 \%$ des membres. Enfin, la croissance du nombre de salariés 
de la Caisse, de 2 à l'origine pour atteindre 25, dont 5 dans les antennes décentralisées, a suivi celle de l'activité de la Caisse.

La Caisse de la culture opère trois points de service, soit celui dédié aux artisans de la culture ainsi que ceux situés dans les locaux de l'UQAM et de l'ONF. $\mathrm{Au}$ plan organisationnel, outre le conseil d'administration, on a créé deux comités d'usagers formés respectivement de membres de l'UQAM et de l'ONF et ce, dans un souci de décentralisation. Un certain pouvoir décisionnel a été conféré à ces comités afin qu'ils puissent apporter une réponse locale à certains enjeux qui leurs sont spécifiques. Ces comités d'usagers peuvent à la fois faire état de demandes particulières, mais aussi adapter certains produits.

Dans les paragraphes qui suivent, nous présentons les éléments marquants du modèle d'intervention de la Caisse de la culture.

\section{La nature intangible du produit fait en sorte qu'elle débourse tous les frais avant même d'être payée.}

\section{Comprendre et accompagner; accompagner pour comprendre}

Les spécificités du secteur culturel évoquées précédemment ne sont pas sans générer des situations et des besoins particuliers en matière de services financiers pour les créateurs, les artistes et les entreprises de ce secteur. En outre, le profil financier de l'artiste moyen, ou du moins la perception qu'en ont les institutions financières, n'est pas sans constituer un frein dans sa recherche de financement, que ce soit à des fins personnelles ou professionnelles. Pour ces raisons, le premier besoin de cette clientèle est tout simplement l'accès à des services financiers de base rendus de façon compétente et professionnelle, adaptés à leur réalité et offert à un coût (taux) concurrentiel. Ainsi, par exemple, on accepte de traiter des demandes de prêts inférieures à $5000 \$$, alors que ce type de produit est considéré comme non rentable par les institutions bancaires. Pourtant, ce type de prêt répond aux besoins d'une très large proportion de la clientèle artistique. En outre, leur condition d'artiste les exclurait du crédit s'ils devaient procéder par le système d'évaluation automatique mis en place par certaines institutions bancaires pour effectuer de tels prêts.

L'impact des spécificités du statut de créateur prend également plusieurs formes. En raison de son statut de travailleur autonome, lorsque le client individuel s'adresse à la Caisse, ce peut être pour des besoins financiers d'ordre personnel (prêt personnel, hypothèque, etc.) ou professionnel (achat d'équipement, d'instruments de musique, de costumes, etc.). En réalité, toutefois, ces deux types de besoins financiers ne sont pas indépendants : des actifs personnels peuvent être utilisés à des fins professionnelles, alors même que des revenus non professionnels peuvent l'être à des fins de dépenses professionnelles. Aussi, l'interprétation des postes financiers de même que la composition des ratios financiers varie selon qu'il s'agit d'un service financier répondant à des besoins personnels ou professionnels. De surcroit, dans le second cas, les méthodes d'analyse se rapprochent davantage de celles utilisées pour une entreprise. De façon à refléter la réalité du travailleur de la culture, et parce que ces deux statuts sont intimement liés, l'analyse que font les conseillers de la Caisse tient compte de ces distinctions, mais également des liens ou des synergies possibles entre les deux types de statut, du point de vue financier. Une telle préoccupation à concilier les deux statuts dans l'analyse peut souvent faire la différence entre l'acceptation ou le rejet d'un dossier, qu'il soit de nature personnelle ou professionnelle.

Du côté de l'entreprise culturelle, nous avons évoqué précédemment ses spécificités du point de vue économique. En outre, la présence d'actifs intangibles, telle la propriété intellectuelle, de même que leur caractère fortement subventionné, tendent à les défavoriser face à des méthodes d'analyse financière standards : alors qu'on juge la santé globale d'une organisation notamment sur la base de son fonds de roulement, cet indicateur n'a aucune valeur dans le cas de l'entreprise culturelle. La nature intangible du produit fait en sorte qu'elle débourse tous les frais avant même d'être payée. Dans ces circonstances, l'absence de fonds de roulement ne donne aucune indication quant à la fragilité globale de l'organisation.

Cette capacité de la Caisse à faire une analyse fine intégrant les spécificités de l'activité du travailleur et de l'entreprise culturelle, et permettant, plus généralement, d'accepter des dossiers qui seraient refusés chez 
le banquier traditionnel, repose sur un ensemble de choix et de politiques organisationnels qui ont été faits très tôt dans la vie de l'organisation.

Une de ces politiques concerne le profil de recrutement des conseillers. Puisqu'il avait été clairement établi, par les fondateurs mêmes de la Caisse, que les artistes vivaient dans leur relation avec leur banquier une non-reconnaissance de leur potentiel et de leurs problématiques se soldant en une forme d'exclusion, il allait de soi qu'on viserait l'embauche de personnes clairement capables de faire le pont entre les besoins et les prérogatives de la clientèle et les contraintes financières de l'institution. À cet égard, le conseiller doit combiner des compétences en analyse financière avec une connaissance et un intérêt pour le milieu culturel. De plus, dans le cours de ses activités, le conseiller est très fortement encouragé à compléter ses connaissances sur le secteur de la culture et ses intervenants et à développer son réseau dans la communauté artistique et des communications. Les conseillers participent activement aux événements culturels. Cette incursion dans l'univers de travail de leurs clients leur permet de mieux en comprendre les particularités, mais également d'aller à la source de certaines informations pouvant leur être utiles dans l'analyse des projets.

Le double profil de financier et de passionné de produits culturels du conseiller se complète d'une préoccupation pour l'établissement de rapports humains avec ses clients et de respect envers le travailleur de la culture. Il ne faut pas perdre de vue que l'artisan de la culture qui s'adresse à la Caisse a souvent essuyé de nombreux refus de la part d'autres institutions financières. De plus, l'écart de valeurs qui caractérise leurs univers respectifs n'est pas sans susciter une grande méfiance de la part des acteurs du milieu culturel face au banquier. Aussi, l'approche personnalisée du conseiller ainsi que sa capacité à comprendre la situation spécifique du client favorisent l'établissement d'un climat de confiance réciproque. La transparence dans les relations qui en résulte permet au conseiller d'intégrer dans son analyse des aspects qualitatifs.

De façon à accentuer cette relation de confiance entre le conseiller et le client (individu ou entreprise), chacun d'eux est attitré à un conseiller et ce, indépendamment de la hauteur de son portefeuille. S'établit ainsi une relation de proximité durable qui bénéficie, au final, au client en raison de la flexibilité qu'elle permet d'introduire dans l'analyse du projet/client. Du fait de la nature de leurs besoins financiers, les travailleurs autonomes peuvent être suivis par deux conseillers (particulier et entreprises) qui travaillent en concertation, ce qui accroît également la stabilité dans les relations entre la Caisse et ses clients.

\section{On accompagne le client dans ses démarches pour l'avancement de son projet en le faisant cheminer à coup de questions et d'entretiens et en cherchant avec lui d'autres partenaires.}

Les dirigeants de la Caisse ont rapidement compris que, pour réussir à concrétiser les financements auprès de leur clientèle, individuelle ou entreprise, il leur faudrait développer un important volet d'accompagnement de celle-ci. Cette approche vise à combler le déficit d'expertise gestionnaire qui caractérise une forte proportion des entreprises culturelles et un certain "analphabétisme économique et financier», voire une aversion pour la chose financière, chez l'artisan de la culture. Le processus d'accompagnement développé à la Caisse prend des formes différentes selon qu'il s'agit de clients particuliers ou d'entreprises culturelles. Dans les deux cas, toutefois, cette intense activité d'accompagnement a pour logique de valoriser le projet client, voire d'accroître sa viabilité financière et, du coup, de diminuer la teneur en risque du projet/client pour la Caisse. Ainsi, du côté des particuliers, les conseillers assument un rôle d'éducation financière autant auprès des petits que des gros comptes. En outre, la Caisse offre la possibilité aux membres de l'UDA qui le souhaitent de déposer leurs cachets en provenance de leur syndicat directement dans des comptes dédiés (impôt, REER, placements, etc.), facilitant ainsi leur planification financière. Le conseiller appuie alors le membre dans sa planification financière. Ce rôle est particulièrement visible lorsqu'un client décroche un important contrat. Comprenant finement le «cycle financier de l'artiste », le conseiller est à même de proposer des conseils adaptés à la réalité de cette profession. L'objectif premier de la démarche n'est pas ici de vendre des services, mais bien de sensibiliser et d'éduquer.

On accompagne le client dans ses démarches pour l'avancement de son projet non pas simplement en exigeant de lui un plan d'affaires, comme c'est la pra- 
tique normale, mais plutôt en le faisant cheminer à coup de questions et d'entretiens et en cherchant avec lui d'autres partenaires pour le financement lorsque le montant l'exige. Si le projet a été sculpté par l'interaction plus rapprochée et intense entre le client et le conseiller, il sera plus naturel pour le conseiller de s'en porter garant auprès de ses collègues d'autres institutions financières, si cela s'avère nécessaire. Il deviendra ainsi le promoteur du projet. Cette approche n'est d'ailleurs pas sans renforcer la fidélité les clients envers l'institution financière.

L'accompagnement de l'entreprise culturelle permet également de développer les capacités administratives de leurs gestionnaires. Des rencontres de formation à la gestion (ex. : à la comptabilité par projet) organisées par les conseillers de la Caisse et un suivi mensuel des états financiers des entreprises clientes visent notamment à outiller et à autonomiser les gestionnaires de ces organisations, libérant d'autant le conseiller pour d'autres clients dans le besoin. De façon plus importante encore, plus l'expertise gestionnaire du client se développe, plus la Caisse est en mesure de travailler en partenariat avec lui afin de développer des solutions sur mesure, sans pour autant accroître son niveau de risque.

\section{Moduler le financement en s'appuyant sur le réseau de partenaires}

Au cours des années, la Caisse a su développer une approche originale du financement. Celle-ci s'appuie très largement sur un réseau de partenaires (financiers ou syndicaux, spécifiques au secteur ou non, collectifs, gouvernementaux ou à but non lucratif) qu'elle a su mobiliser autour des besoins de ce milieu. Ce travail partenarial prend diverses formes.

Le partenariat fondateur avec l'UDA s'est rapidement concrétisé dans le développement d'une offre de service spécifique. Les contrats-UDA en sont l'exemple même. Dès qu'un comédien obtient un contrat, la Caisse peut mettre le montant correspondant à sa disposition sur la simple base de la garantie que constitue un contrat signé selon les conventions de l'UDA avec les producteurs. Cette disposition est à ce point avantageuse pour les membres de l'UDA que celle-ci a intégré à son offre les services de la Caisse.

Il en est de même de l'entente partenariale développée au cours des dernières années entre le CALQ
(Conseil des arts et des lettres du Québec ${ }^{4}$ ), la SODEC (Société de développement des entreprises culturelles $^{5}$ ) et la Caisse de la culture. Jusque là, les subventions et garanties de prêts octroyées par le CALQ étaient déboursées par la SODEC. Ce processus de gestion quotidienne des subventions était considéré très lourd et occasionnait des délais importants de versement de la subvention. Or, les organisations culturelles se caractérisant par un manque structurel de fonds de roulement, ces délais entraînaient, trop souvent, l'abandon de projets. Aujourd'hui, la Caisse de la culture s'est substitué à la SODEC en versant la subvention dès le moment où le CALQ émet un avis favorable et ce, même si l'organisation bénéficiaire n'a pas encore reçu le montant octroyé. Ce prêt-relais peut devancer même d'une année le versement réel de la subvention.

Ce type d'entente, selon laquelle la Caisse avance des fonds à des clients sur la base d'une simple lettre de confort émise par l'agence gouvernementale, n'aurait pu se concrétiser sans le développement préalable d'une forte relation de confiance entre les partenaires qui repose sur des années de collaboration dans le soutien au développement du secteur culturel. Avec le temps, les partenaires ont appris à connaître leur métier et leurs capacités respectives d'intervention. Pour les organismes publics, la Caisse est la seule institution financière qui ait la connaissance du secteur et l'ouverture permettant de développer de telles ententes. Aussi, on lui a accordé l'exclusivité de ces prêtsrelais. Du point de vue de la Caisse, cette entente a permis de développer de façon importante sa clientèle entreprise qui doit obligatoirement détenir un compte chez elle pour bénéficier de cet avantage.

La Caisse est également proactive dans le développement de nouveaux partenariats visant à enrichir le portefeuille de produits et services financiers accessibles aux artisans de la culture. Elle a été l'initiatrice d'un partenariat innovateur avec Exportation et Développement Canada (EDC) qui offre notamment une aide pré-exportation. Ce programme, jusque là utilisé essentiellement dans le cas d'activités manufacturières impliquant une production de biens tangibles, permet de financer la préparation et la livraison à l'étranger du produit, remboursé ensuite par les produits générés par le contrat ${ }^{6}$. Après plus de cinq mois d'échanges, ce programme peut dorénavant bénéficier aux entreprises culturelles qui souhaitent faire le saut sur les marchés étrangers. Elle a également su mobiliser 
Investissement Québec (IQ), et son offre de produits, autour des dossiers d'entreprises culturelles à statut d'OBNL (Organismes à but non lucratif), alors que cette société d'État n'avait jusque là qu'une connaissance très limitée des spécificités de l'entreprise culturelle.

Plus globalement, cette approche résolument proactive de la Caisse de la culture dans le développement de partenariats avec des organisations offrant des produits financiers complémentaires s'inscrit dans une approche de modulation de l'offre financière à sa clientèle. Au fil des années, les institutions de financement impliquées dans la culture ont développé nombre de programmes d'aide et de support pour les entreprises du secteur culturel, parfois en partenariat, de sorte que ces mesures, en se complétant, constituent maintenant une toile de plus en plus solide permettant à la plupart des projets valables d'avoir une chance de voir le jour et ce, en dépit des risques et des préjugés. En mobilisant plusieurs partenaires financiers autour d'un projet, la Caisse est aujourd'hui en mesure de proposer à son entreprise cliente une solution adaptée à ses besoins à court terme et lui permettant d'envisager l'ensemble de son financement dans une perspective de long terme. Une telle démarche d'ingénierie financière serait impensable sans un travail intensif avec des partenaires de confiance.

\section{Développer des méthodes d'analyse et des approches de gestion du risque adaptées}

Un des enjeux importants que rencontre une organisation comme la Caisse de la culture est celui de concilier les impératifs de rentabilité à ceux de services à des membres qui sont souvent exclus de l'offre des banques traditionnelles. Dans le cas d'une institution financière, la rentabilisation de l'activité passe par une capacité à contrôler les risques. À cet égard, deux processus organisationnels sont particulièrement centraux, soit l'évaluation des dossiers ainsi que la gestion du risque global de la Caisse.

Une des principales difficultés que présente le milieu culturel pour l'analyste financier est le caractère intangible du produit qui se traduit, à toute fin pratique, par une absence de matières premières et d'inventaire, et donc de garanties tangibles. Au caractère intangible du produit s'ajoute le fait que le cycle de production culturel implique que de lourds investissements soient faits souvent longtemps avant qu'ils ne génèrent de revenus. C'est sans compter que la demande pour le produit culturel est davantage aléatoire que pour un produit dont on peut lancer un prototype avant sa finalisation afin d'évaluer son marché potentiel. Bien sûr, un producteur et une équipe d'expérience et de renom ayant déjà engrangé plusieurs succès présenteront un moindre risque et auront plus facilement accès au crédit. Les créateurs plus jeunes sont de ce fait considérés comme présentant un niveau de risque supérieur. L'évaluation juste d'un projet culturel requiert une connaissance fine et approfondie des spécificités de l'activité de l'artisan culturel et le développement de méthodes appropriées.

Face à ces écueils, la Caisse de la culture a, au cours des années, développé un ensemble d'outils, d'approches et de méthodes permettant de tenir compte de ses spécificités. Le ratio de capacité de remboursement est, par exemple, calculé de façon à prendre en compte les chevauchements entre dépenses de consommation et d'exploitation qui caractérisent l'activité du travailleur de la culture, accroissant du coup le revenu disponible et la capacité d'emprunt. De même, l'évaluation des revenus futurs de l'artisan culturel étant fortement aléatoire, la Caisse de la culture a donc mis sur pied une méthode alternative basée non pas sur les revenus anticipés, mais plutôt sur les revenus passés.

\section{Une telle démarche d'ingénierie financière serait impensable sans un travail intensif avec des partenaires de confiance.}

Certains conseillers vont même jusqu'à avancer qu'une fois les postes comptables et les ratios réinterprétés en fonction des spécificités de ce secteur d'emploi, l'activité culturelle ne présente souvent pas plus de risque que d'autres secteurs. Selon eux, une analyse financière tenant compte de ces paramètres permet d'observer une génération de valeur, par l'entreprise culturelle, bien davantage supérieure à celle tirée d'une interprétation financière plus classique.

Au-delà de l'évaluation financière du projet, qu'il soit celui d'un individu ou d'une entreprise, la Caisse intègre à son analyse un volet qualitatif non négligeable. Cette approche vise à prendre en compte des facteurs intangibles susceptibles de contribuer à la valeur du projet. Ces facteurs intangibles sont caractéristiques d'une économie de la culture fondée, pour reprendre l'approche des conventions, sur le «renom» et le 
« projet $»^{7}$. La manière dont le projet est perçu par les pairs et la capacité du promoteur à bénéficier de ressources partenariales apparaissent souvent déterminantes de sa réussite future.

Aux yeux de ce banquier, le promoteur du projet est susceptible de constituer le plus important actif intangible. C'est pourquoi, avant même d'analyser les chiffres, le conseiller accorde une attention particulière aux motivations du demandeur. Comme le note un conseiller de la Caisse : " On se fie aussi beaucoup au dynamisme, à la détermination et au degré de foi dans leur projet que les entrepreneurs peuvent démontrer. Parfois, après seulement dix minutes de discussion avec celui-ci, on sait si on croit ou non en son potentiel, si on va faire le financement et ce, avant même d'avoir vu les chiffres. Après ça, on lui dit: "Je vais $m$ 'arranger avec la poutine pour te trouver des garanties". Et si jamais il y a un manque de garanties, c'est là qu'on va se retourner vers la SODEC, le ministère de la Culture ou autres partenaires financiers afin de lui obtenir de l'aide de façon à ce que le projet se concrétise ». Évidemment, l'expérience du conseiller ainsi que sa connaissance du milieu sont des atouts incontournables dans l'évaluation des intangibles de cette nature, sans compter le bassin informationnel que constitue le réseau de partenaires qui a souvent des antécédents de travail avec les artisans qui se présentent à la Caisse dans le but d'obtenir un financement.

\section{Aux yeux de ce banquier, le promoteur du projet est susceptible de constituer le plus important actif intangible.}

Le réseau de partenaires joue un rôle central dans la capacité de la Caisse à apporter une solution à ses membres. À défaut de pouvoir s'appuyer sur des garanties tangibles sous forme d'inventaires, d'actifs immobiliers ou autres, la Caisse s'appuie, dans son offre, sur les garanties intangibles offertes par les institutions partenaires. Ces garanties prennent la forme du contrat UDA, de la garantie pré-exportation d'EDC dont nous avons parlé plus tôt, des prêts garantis d'IQ, du prêt-relais de la SODEC. À la lumière de sa connaissance du secteur de la culture et du suivi de son évolution qu'elle en fait, la Caisse joue un rôle proactif dans le développement du réseau de partenaires susceptibles d'apporter une réponse à de nouveaux besoins financiers des artisans du secteur.
La diversité de ces partenaires, tant en termes de métiers (bancaire, financier de capital de risque, défense de travailleurs, exportation, protection et développement de culture, etc.) que de gouvernance (coopérative, ministère, entreprise d'état, syndicat), n'est pas toutefois sans porter les germes d'interprétation différenciée de la qualité d'un projet. La confiance qui s'est installée entre les partenaires au cours des années est fondamentale pour que la complémentarité entre les acteurs prenne le dessus sur les divergences potentielles. Comme le note une conseillère de la Caisse, de tels partenariats pourraient difficilement se concrétiser au niveau fédéral. Des expériences antérieures de cette nature se sont en effet soldées par des pertes pour la Caisse, minant les relations et la confiance envers certains acteurs gouvernementaux du milieu culturel canadien, en l'occurrence.

C'est également par ses nombreux partenariats dans les montages financiers que la Caisse, ainsi que ses partenaires, parvient à partager son risque d'affaires. Encore là, la connaissance réciproque des partenaires, en dépit de leur diversité, et la confiance qui caractérise leurs rapports favorisent une approche plus transparente qui, parce qu'elle participe à réduire le « risque partenarial », permet d'aller plus loin dans l'offre de financement aux créateurs.

La diversification du risque prend, pour sa part, une forme bien particulière à la Caisse de la culture. En effet, en plus de la modulation de l'offre et des partenariats touchant les fournisseurs et les compétiteurs, la Caisse de la culture a cherché à réduire son risque en augmentant ses capacités financières grâce à la diversification prudente des clientèles. Elle procédait ainsi au développement d'un centre complet de services à l'UQAM, augmentant la clientèle tout en la diversifiant. En intégrant la clientèle des travailleurs de l'UQAM (professeurs, employés de soutien, professionnels, etc.) à la sienne, la caisse ajoutait une clientèle salariée susceptible de générer de l'épargne.

\section{Conclusion}

Dans cette étude, nous avons souhaité mettre en évidence la capacité de la forme coopérative à s'adapter à des territoires qui lui sont nouveaux et ce, en prenant à témoin le secteur financier. Lorsque l'acteur financier joue pleinement son rôle, il est appelé à jouer un rôle central de levier dans le développement social et économique de territoires, et encore davantage lors- 
qu'il s'agit de territoires marginalisés. Le secteur culturel québécois constitue un de ces territoires périphériques dans ce grand ensemble en voie de mondialisation qu'est l'industrie culturelle. Vivre de la culture, au Québec, est encore réservé à une très faible minorité. L'action de la Caisse Desjardins de la Culture s'inscrit spécifiquement dans ce contexte d'une trop fréquente exclusion des artisans québécois de la culture de ce levier fondamental qu'est l'activité financière.

Le cadre d'analyse des ressources que nous avons adopté nous permet de retracer certaines des conditions par lesquelles un acteur financier comme la Caisse Desjardins de la culture en arrive à se positionner comme central dans le développement d'un territoire marginalisé, tout en assurant sa viabilité à long terme dans un univers financier dont les règles et les normes laissent de moins en moins de marge de manœuvre. La résolution de cette équation complexe passe par le développement d'un modèle organisationnel traversé par l'innovation.

\section{La capacité à développer des relations de confiance avec des partenaires du milieu apparaît également comme une condition de succès incontournable.}

La forme coopérative adoptée, dès sa création, par les acteurs du milieu pour développer une banque qui soit à leur service constitue aujourd'hui un de ses actifs les plus précieux. En outre, en adoptant la loupe des processus organisationnels, nous avons pu mettre en évidence les relations entre la propriété collective et la capacité d'adaptation aux besoins du milieu. La capacité à faire et développer des partenariats durables avec ce milieu n'est pas la moindre. La compatibilité des visions de développement à long terme, tant avec les partenaires sectoriels que financiers, anciens et récents, a notamment permis d'asseoir des relations de confiance qui bénéficient à terme aux artisans du secteur au service desquels ils se mettent. La relative autonomie dont elle bénéficie, en comparaison à la succursale bancaire, et les méthodes et outils d'analyse financière sur mesure des projets que cette décentralisation décisionnelle permet notamment de développer expliquent largement sa capacité à mettre en œuvre son propre projet organisationnel. De même, l'affirmation très claire, au départ, d'une mission de développement du milieu de la culture, plutôt qu'une simple réponse aux besoins des sociétaires, prend racine dans sa gouvernance même, alors que les acteurs du milieu participent intensivement aux instances décisionnelles démocratiques.

Si la forme coopérative apparaît comme condition première de la capacité de cette institution financière à participer activement au développement du milieu québécois de la culture, elle ne s'avère toutefois pas suffisante. En effet, il apparaît que la recherche d'équilibre traverse également l'ensemble des choix de développement de cette organisation. Le développement du secteur entreprises et l'ouverture à d'autres types de clientèles, milieux et segments (musiciens, écrivains, journalistes, enseignants, voire maintenant les professionnels du cinéma) se font progressivement, au fur et à mesure que l'organisation construit et consolide ses compétences. Au plan structurel, cette diversification de la clientèle, et plus spécifiquement l'intégration d'une clientèle salariée non issue du secteur de la culture, s'accompagne d'une certaine décentralisation décisionnelle, tant au plan opérationnel que démocratique.

La capacité à développer des relations de confiance avec des partenaires du milieu apparaît également comme une condition de succès incontournable. L'intégration de son réseau de partenaires à ses activités montre une forme moderne de fonctionnement en réseau aussi bien sur une base stratégique qu'opérationnelle, alors que les méthodes de travail sont teintées de l'ouverture de l'organisation sur son milieu. Ainsi, la qualité de la relation, le degré de détail des informations recueillies, la confiance qui s'établit de même que les moyens de vérification et de contrôle qu'apportent la participation et l'interaction avec le réseau expliquent notamment les performances remarquables de la Caisse en termes de fidélisation, de minimisation des défauts de paiements, de croissance générale et de contribution au milieu. Le réseau de partenaires de la Caisse constitue aujourd'hui un de ses actifs les plus précieux.

La Caisse de la culture apparaît donc exemplaire de la manière dont l'organisation coopérative peut constituer une forme innovante affirmant la capacité de laboratoire propre à ce type d'organisation. De plus, l'étude empirique présentée met en évidence la capacité de l'organisation coopérative évoluant en contexte de forte pression des marchés de maintenir une mission tant d'intérêt général que collectif. Cette ca- 
pacité, fondamentale pour le développement des milieux, est toutefois conditionnelle à une volonté affirmée d'innovation continue dans le modèle organisationnel.

\section{Notes et références}

1 Grant, R. M. (1991). «The Resource-Based Theory of Competitive Advantage : Implications for Strategy Formulation ", California Management Review,vol. 91, $\mathrm{n}^{\mathrm{o}}$ 3, p. 481-510; Wernerfelt, B. (1984). «A Resource-Based View of the Firm », Strategic Management Journal, vol. 5, $\mathrm{n}^{\mathrm{o}}$ 2, p. 171-180; Vézina, M. (2000). «L'approche des ressources : un nouveau paradigme pour la réflexion stratégique », dans T. Hafsi et al., La stratégie des organisations : une synthèse, éd. Transcontinental Inc.

2 Ainsi selon le «portrait socio-économique des artistes » édité par le ministère de la Culture et des Communications, $29 \%$ des artistes connaissent de grandes fluctuations de revenus de l'ordre de $50 \%$ d'une année à l'autre; $44 \%$ des artistes gagnent moins de $20000 \$$ et se partagent $11,5 \%$ de la masse totale des revenus; $22,4 \%$ déclarent des revenus de plus de $50000 \$$ et se partagent $60 \%$ de la masse totale des revenus. Voir MCCQ (2004). «Pour mieux vivre de l'art: portrait socioéconomique des artistes », Gouvernement du Québec, février (disponible sur www.mcc.gouv.qc.ca ).

3 Selon D. St-Pierre, la reconnaissance d'une multiplicité de pôles légitimes (État, société civile, professionnels du secteur, autres organisations collectives) peut être vue comme une particularité forte du Québec par rapport aux autres pays. La France est, à titre d'exemple, un cas de co-gestion entre État et professionnels où la société civile et les autres organisations collectives sont beaucoup moins présentes. Voir St-Pierre, D. (2003). La Politique culturelle de 1992 : continuité ou changement?, Presses de l'Université Laval, collection Management public et gouvernance.

4 Le CALQ est une société d'État qui se consacre au développement et à la diffusion des arts. C'est par son entremise que le gouvernement québécois offre son soutien financier aux artistes professionnels et aux organismes artistiques sans but lucratif.

5 La SODEC est une société d'État relevant de la ministre de la Culture et des Communications. Son mandat est de promouvoir et de soutenir dans toutes les régions du Québec l'implantation et le développement des entreprises culturelles. Pour ce faire, elle met à la disposition des entreprises culturelles différents produits financiers tels garanties, prêts, crédit rotatif, capital-actions. La SODEC agit également comme gestionnaire des crédits d'impôts du Québec pour certains secteurs culturels.

6 Le produit offert par EDC (le « financement pré-expédition ») ressemble un peu à une marge de crédit offerte sur la base du contrat pour certains types de dépenses précises telles que la production et la livraison. EDC offre une garantie à hauteur de $75 \%$, la Caisse de la Culture prenant le risque complémentaire $(25 \%)$.

7 Boltanski, L. et E. Chiapello (1999). Le Nouvel Esprit du Capitalisme, Gallimard, NRF Essais. 


\section{Publicité}

Maîtrise en gestion de projet 